\title{
Coronavirus Disease in South Korea: Antibody Testing from the Perspective of Effective Quarantine Measures
}

\author{
Insuk Sim ${ }^{1}$ and Yun-Jung Kang ${ }^{2 *}$ (iD \\ ${ }^{1}$ Department of Clinical Laboratory Science, Kyungdong University, Wonju 26495, Republic of Korea. \\ ${ }^{2}$ Department of Clinical Laboratory Science, Ansan University, Ansan 15328, Republic of Korea.
}

\begin{abstract}
The coronavirus disease (COVID-19) pandemic, which began in December 2019, spread rapidly across Asian countries in January and February 2020 and again after March 2020. COVID-19, caused by severe acute respiratory syndrome coronavirus 2 (SARS-CoV-2), is characterized by fever, cough, and dyspnea. On January 31, 2020, the World Health Organization declared the Public Health Emergency of International Concern. Quarantine authorities are constantly working to prevent the spread of COVID-19. One of the control measures is preparing for national antibody testing, as another wave of infection is expected to occur in the fall of 2021. There are three reasons for antibody testing from a prevention perspective. First, it can identify people with asymptomatic infection. Second, it can detect whether neutralizing antibodies are produced in individuals who have already been infected. Third, it can confirm collective immunity at a community or population level. Considering the lack of effective antiviral drugs or vaccines, the strategy of implementing an effective antibody testing program is an important control measure to minimize the damage caused by the COVID-19 pandemic.
\end{abstract}

Keywords: Antibody test, Asymptomatic infection, Collective immunity, COVID-19, SARS-CoV-2, Neutralizing antibody

\footnotetext{
*Correspondence: Ivpig@naver.com

(Received: December 24, 2020; accepted: March 16, 2021)

Citation: Sim I, Kang Y-J. Coronavirus Disease in South Korea: Antibody Testing from the Perspective of Effective Quarantine Measures. J Pure Appl Microbiol. 2021;15(2):587-589. doi: 10.22207/JPAM.15.2.12

(C) The Author(s) 2021. Open Access. This article is distributed under the terms of the Creative Commons Attribution 4.0 International License which permits unrestricted use, sharing, distribution, and reproduction in any medium, provided you give appropriate credit to the original author(s) and the source, provide a link to the Creative Commons license, and indicate if changes were made.
} 
The coronavirus disease (COVID-19) pandemic, which began in December 2019 in Wuhan, China, spread rapidly across Asian countries in January and February 2020 and again after March 2020. Coronaviruses are frequent causes of respiratory infections, and six major species, besides severe acute respiratory syndrome coronavirus 2 (SARS-CoV2), are known to cause human infections. These species include highly pathogenic severe acute respiratory syndrome coronaviruses, Middle East respiratory syndrome coronavirus, and four less virulent species, namely NL63, 229E, OC43, and HKU14 ${ }^{1}$. COVID-19 is characterized by fever, cough, and dyspnea ${ }^{2}$. After human-to-human transmission was confirmed, containment measures were implemented to limit the movement of people between the cities of Wuhan and Hubei. These phenomena resulted in a spillover effect observed in the occurrence of common infectious diseases. SARS-CoV-2, in keeping with most respiratory viruses, is considered to be primarily transmitted directly via droplets from infected people or indirectly via contact with fomites in a contaminated environment. On January 31, 2020, the World Health Organization (WHO) declared the Public Health Emergency of International Concern ${ }^{3}$. However, by March 10, 2020, there were approximately 118,000 patients and 4,200 deaths worldwide, mainly in East Asia, Europe, and North America. Therefore, the WHO announced a pandemic on March 11, 20204. Individuals with SARS-CoV-2 infection develop symptoms of upper respiratory tract infection with fever after an average incubation period of approximately 5 to 6 days. The maximum incubation period is 14 days, and the probability of symptom onset after more than 14 days is approximately $0.01 \%{ }^{5}$. Approximately $80 \%$ of infected individuals develop mild upper respiratory tract infections or mild-to-moderate pneumonia. About $14 \%$ of infections result in severe disease with dyspnea and hypoxia, and about $5 \%$ of infections result in critical diseases with complications such as respiratory failure, shock, and multiple organ damage $^{6}$. In Korea, since the first patient was diagnosed with SARS-CoV-2 infection on January 20,2020 , the number of infected patients in the Sincheonji area in Daegu has rapidly increased ${ }^{7}$, although it is currently stable. As of December
24,2020 , the cumulative number of confirmed patients was 53,533 , and the number of deaths was 756. Quarantine authorities are constantly working to prevent the spread of COVID-19. One of the measures is preparing for a national antibody test because a wave of infection is expected to occur again in the winter of 2021/2022. We analyzed blood samples from 1,500 Daegu and Gyeongsan residents. Among these, 500 ages treated with COVID-19 and 1,000 were noninfected. In addition, 7,000 blood samples were obtained and tested nationwide. Antibody tests were used to test for SARS-CoV-2 antibodies in the blood, unlike the commonly used SARS-CoV-2 RNA tests. If antibodies are present, it can be assumed that the individual has been immunized against the disease and has been infected in the past. There are three reasons for antibody testing from a prevention perspective. First, it identifies individuals with asymptomatic infection. The incidence of asymptomatic infection varies depending on the scope of environmental and epidemiological investigations of the outbreak. For example, in the COVID-19 epidemic on board the Diamond Princess cruise ship, 51\% of the confirmed cases were asymptomatic when mass screening was conducted, regardless of the presence of symptoms ${ }^{8}$. In Italy, $44 \%$ of individuals diagnosed with SARS-CoV- 2 infection were asymptomatic, and in Japan, $0.06 \%$ of individuals diagnosed with the infection were asymptomatic ${ }^{9}$. In China, $75 \%$ of patients who were asymptomatic at the time of diagnosis developed symptoms later, and $1-3 \%$ of patients were known to have persistent asymptomatic infection. Individuals with COVID-19 present with very few initial symptoms, although they are capable of transmitting the virus during the prodromal phase. Overall, approximately $80 \%$ of individuals with COVID-19 have mild symptoms and recover naturally, although in adults aged over 60 years or those with chronic underlying diseases, symptoms tend to worsen 7-9 days after the onset, with the occurrence of pneumonia and acute respiratory distress syndrome. An antibody test is used to identify individuals with asymptomatic infection. Antibody testing can identify asymptomatic individuals early and prevent the spread in the community. Moreover, identifying individuals with asymptomatic past infection provides information 
on the characteristics of individuals who are predominant spreaders and the characteristics of individuals who are likely to recover without treatment. Identifying such cases makes it possible to establish effective control measures. Second, antibody testing determines whether neutralizing antibodies were produced. Enhancing antibodies are antibodies that prevent visceral invasion. Whether or not antibodies are formed is important, although it is necessary to analyze the ratio of the number of neutralizing antibodies formed among the antibodies to proceed with an immunological study. Antibody research can also help develop vaccines and other measures to prevent reinfection. Third, antibody testing can confirm collective immunity. Collective immunity is a phenomenon in which most of a population is immune to an infectious disease, and this provides indirect protection to those who are not immune to the disease. Through collective immunity, people with poor immunity and older adults with various comorbidities can be protected. Experts predict that approximately $60 \%$ of the population will not become reinfected if antibodies are produced after infection. Therefore, it is possible to determine how well a member of a group has been immunized against COVID19 and to adjust the stage of prevention. Considering the lack of effective antiviral drugs or vaccines, the strategy of establishing an effective antibody testing program is an effective control measure that is currently available that can minimize the impact of the COVID-19 epidemic.

\section{ACKNOWLEDGMENTS}

This work was supported by Basic Science Research Program through the National Research Foundation of Korea (NRF).

\section{CONFLICT OF INTEREST}

The authors declare that there is no conflict of interest.

\section{FUNDING}

This work was funded by the Ministry of Science and ICT (No. 2018R1C1B5086313).

\section{AUTHORS' CONTRIBUTION}

YJK and ISS wrote the entire manuscript Both the authors read and approved the manuscript for publication.

\section{DATA AVAILABILITY}

The datasets used and/or analyzed during the current study are available from the corresponding author on reasonable request.

\section{ETHICS STATEMENT}

Not applicable.

\section{REFERENCES}

1. Su S, Wong G, Shi W, et al. Epidemiology, genetic recombination, and pathogenesis of coronaviruses. Trends Microbiol. 2016;24(6):490-502. doi:10.1016/j. tim.2016.03.003

2. Huang C, Wang Y, Li X, et al. Clinical features of patients infected with 2019 novel coronavirus in Wuhan, China. The Lancet. 2020;395(10223):497-506. doi: 10.1016/ S0140-6736(20)30183-5

3. Chan JF, Yuan S, Kok KH, et al. A familial cluster of pneumonia associated with the 2019 novel coronavirus indicating person-to-person transmission: a study of a family cluster. Lancet. 2020;395(10223):514-523. doi: 10.1016/S0140-6736(20)30154-9

4. World Health Organization. WHO director-general's opening remarks at the media briefing on COVID-19 [Internet]. Geneva $(\mathrm{CH})$ : World Health Organization, c2020 [cited 2020Mar 15]. https://www.who.int/dg/ speeches/detail/who-director-general-s-openingremarks-at-the-mediabriefing-on-covid-19---11march-2020.

5. Lauer SA, Grantz KH, Bi Q, et al. The incubation period of coronavirus disease 2019 (COVID-19) from publicly reported confirmed cases: estimation and application. Annals Intern Med. 2020:172(9):577-582. doi: 10. 7326/M 20-0504

6. Wu Z, McGoogan JM. Characteristics of and important lessons from the coronavirus disease 2019 (COVID-19) outbreak in China: summary of a report of 72314 cases from the Chinese Center for Disease Control and Prevention. JAMA.2020;323(13):1239-1242. doi: 10.1001/jama.2020.2648.

7. Park JY. "Corona Corresponds to Korea, Being Caught by Sinchon and Conservatives". New 1. 2020. https:// www.news1.kr/articles/?3858187 (Korean).

8. Japanese National Institute of Infectious Diseases. Field briefing: diamond princess COVID-19 cases, 20 Feb Update [Internet]. Tokyo (JP): Japanese National Institute of Infectious Diseases. c2020. https://www. niid.go.jp/niid/en/2019-ncov-e/9417-covid-dp-fe-02. html.

9. European Centre for Disease Prevention and Control. Rapid risk assessment: novel coronavirus disease 2019 (COVID-19) pandemic: increased transmission in the EU/EEA and the UK - sixth update [Internet]. Stockholm (SE): European Centre for Disease Prevention and Control. c2020. https://www.ecdc.europa.eu/en/ publicationsdata/ rapid-risk-assessment-novelcoronavirus-disease- 2019-covid-19-pandemicincreased. 